\title{
FOURIER ANALYSIS ON COMPACT SYMMETRIC SPACE
}

\author{
BY THOMAS O. SHERMAN
}

Communicated by R. R. Goldberg, November 5, 1976

1. Let $L \supset K$ be Lie groups with complex Lie algebras $\mathfrak{l}_{c}$ and $\mathfrak{f}_{c}$. Assume $\mathfrak{t}_{c}$ has a linear complement $\mathfrak{b}$ in $\mathfrak{l}_{c}$ which is a subalgebra. For any $\sigma$ in LieHom $_{\mathrm{C}}(\mathfrak{b}, \mathrm{C})$ there is a unique germ of a $\mathrm{C}^{\omega}$ function $e^{\sigma}$ at $s_{0}:=K$ in $S:=$ $L / K$ such that $e^{\sigma}\left(s_{0}\right)=1$ and $x e^{\sigma}=\sigma(x) e^{\sigma}(x$ in $\mathfrak{b})$. Now suppose $S$ is connected, $K$ is compact, and $e^{\sigma}$ extends to an element of $C^{\omega}(S)$. Then (HarishChandra) $\varphi_{\sigma}(s):=\int_{K} e^{\sigma}(k s) d k$ is a spherical function in the sense that

$$
\int_{K} \varphi_{\sigma}(g k s) d k=\varphi_{\sigma}(g K) \varphi_{\sigma}(s) .
$$

For a Riemannian symmetric space of noncompact type Helgason [1], [2] extended Harish-Chandra's spherical transform theory to a Fourier theory in which functions of the form $e^{\sigma}$ mimic the role of characters in classical Fourier theory on $\mathbf{R}^{n}$. Here we report that difficulties inherent in copying these ideas over to compact symmetric space have been overcome, at least for the rank one spaces.

2. Let $S:=U / K$ be symmetric with $U$ compact semisimple. Let $G_{c}$ be a complexification of $U$ and $G$ a noncompact real form of $G_{c}$ such that $K_{0}:=G$ $\cap U$ is open in $K$, and maximal compact in $G$. Let $g=\mathfrak{l}+\mathfrak{a}+\mathfrak{n}$ be an Iwasawa decomposition and set $\mathfrak{b}:=\mathbf{C}(\mathfrak{a}+\mathfrak{n})$. Then $\mathfrak{g}_{c}=\mathfrak{f}_{c}+\mathfrak{b}$ as in $\S 1$. $\Lambda$ will denote the set of those $\lambda$ in LieHom $_{\mathbf{C}}(\mathfrak{b}, \mathbf{C})$ such that $e^{\lambda}$ is in $C^{\omega}(S)$. $\Lambda \mid i \mathfrak{a}$ is the set of highest restricted weights of $K$-spherical representations of $U$. For $\lambda$ in $\Lambda$ let $V_{\lambda}$ denote the corresponding irreducible $U$-submodule of $L^{2}(S)$. Then $e^{\lambda}$ is the highest weight vector in $V_{\lambda}$. Define $\tau$ in $\operatorname{LieHom}_{\mathrm{C}}(\mathfrak{b}, \mathbf{C})$ by $\tau(x):=\operatorname{tr}(\operatorname{ad} x \mid \mathfrak{b})$ $(x$ in $\mathfrak{b})$. Then $\tau$ is in $\Lambda$.

LEMma 1. There is a unique maximal connected, open, $K$-invariant neighborhood $S_{0}$ of $s_{0}$ in $S$ on which $e^{\tau} \neq 0$. Then $e^{\lambda} \neq 0$ on $S_{0}$ for all $\lambda$ in $\Lambda$.

On $S_{0}$ define $e_{*}^{\lambda}:=\left(e^{\lambda+\tau}\right)^{-1}$. $e_{*}^{\lambda}$ is the inverse transform kernel to $e^{\lambda}$. The aforementioned "inherent difficulty" of the subject is the singularity of $e_{*}^{\lambda}$ off of $S_{0}$. Let $B:=K / M$ where $M$ is the centralizer of $\mathfrak{a}$ in $K$.

Lemma 2. For all $u K$ in $S_{0}$, $s$ in $S$, and $\lambda$ in $\Lambda$

AMS (MOS) subject classifications (1970). Primary 43A30, 43A85, 42A76, 22 E30. 


$$
\int_{B} e^{\lambda}\left(k^{-1} s\right) e_{*}^{\lambda}\left(k^{-1} u K\right) d k M=\varphi_{\lambda}\left(u^{-1} s\right) .
$$

Proof. While this result may be proved directly in the full generality of $\S 1$ it follows in the present case by analytic continuation via $G_{c}$ of the similar result of Helgason on $G / K_{0}$ (e.g. middle of p. 116, [1]). [5] contains related analytic continuation arguments and helpful machinery linking $U / K$ and $G / K_{0}$.

Let $L_{c}^{2}\left(S_{0}\right):=\left\{f \in L^{2}\left(S_{0}\right) \mid \operatorname{supp}(f)\right.$ is compact in $\left.S_{0}\right\}$ and let $d_{\lambda}:=$ $\operatorname{dim}\left(V_{\lambda}\right)$. Lemma 2 combines with well-known harmonic analysis on $S$ (see e.g. [3, Chapter 10]) to give

THEOREM 1. For $f_{1}$ in $L_{c}^{2}\left(S_{0}\right)$ define $F_{*} f_{1}$ on $B \times \Lambda$ by

$$
F_{*} f_{1}(k M, \lambda):=\int_{S_{0}} f_{1}(s) e_{*}^{\lambda}\left(k^{-1} s\right) d s .
$$

Then $\Sigma_{\Lambda} d_{\lambda} \int_{B} F_{*} f_{1}(k M, \lambda) e\left(k^{-1} s\right) d k M \rightarrow f_{1}(s)\left(\right.$ in $\left.L^{2}\left(S_{0}\right)\right)$.

THEOREM 2. For $f_{2}$ in $L^{2}(S)$ define $F f_{2}$ on $B \times \Lambda$ by

$$
F f_{2}(k M, \lambda):=\int_{S} f_{2}(s) \text { conj. }\left(e^{\lambda}\left(k^{-1} s\right)\right) d s .
$$

Then $\Sigma_{\Lambda} d_{\lambda} \int_{B} F f_{2}(k M, \lambda)$ conj. $\left(e_{*}^{\lambda}\left(k^{-1} s\right)\right) d k M \rightarrow f_{2}(s)\left(\right.$ in $\left.L^{2}(S)\right)$.

THEOREM 3. For $f_{1}$ in $L_{c}^{2}\left(S_{0}\right), f_{2}$ in $L^{2}(S)$

$$
\int_{S_{0}} f_{1}(s) \operatorname{conj} .\left(f_{2}(s)\right) d s=\Sigma_{\Lambda} d_{\lambda} \int_{B} F_{*} f_{1}(b, \lambda) \operatorname{conj} .\left(F f_{2}(b, \lambda)\right) d b \text {. }
$$

3. To extend these results from $S_{0}$ to $S$ we must give global definitions of $e_{*}$ and $F_{*}$. This is done for $S$ of rank one as follows. Let $\lambda_{1}$ be the generator $\left(\right.$ over $\left.\mathbf{Z}^{+}\right)$of $\Lambda$. Let $\rho:=\operatorname{Re}\left(e^{\lambda_{1}}\right)$ except $\rho:=1$ if $S=P_{d}(\mathbf{R})$. Then

$$
e_{*}^{\lambda}(s):=(\operatorname{sgn}(\rho(s)))^{1+\operatorname{dim} S}\left(e^{\lambda+\tau}(s)\right)^{-1} \quad(s \text { in } S) .
$$

Where $\mu(s)$ is the distance on $S$ from $s_{0}$ to $s$ let

$$
S(\alpha, \beta):=\left\{s \in S|| \rho(s) \mid \geqslant \alpha, \mu(s) \leqslant-\beta+\sup _{x \in S} \mu(x)\right\} .
$$

Then for $f$ in $C^{\infty}(S)$,

$$
F_{*} f(k M, \lambda):=\lim _{\beta \rightarrow 0^{+}} \lim _{\alpha \rightarrow 0^{+}} \int_{S(\alpha, \beta)} f(s) e_{*}^{\lambda}\left(k^{-1} s\right) d s
$$

defines a distribution on $S$, at least for the rank one symmetric spaces, and if we replace $S_{0}$ by $S$ and $L_{c}^{2}\left(S_{0}\right)$ by $C^{\infty}(S)$ in Theorems 1 and 3 they continue to hold. Theorem 2 may also be made global by carefully defining the order of integration over $B$. This has been carried out in detail for the sphere in [4].

\section{REFERENCES}

1. S. Helgason, A duality for symmetric spaces with applications to group representa. tions, Advances in Math. 5 (1970), 1-154. MR 41 \#8587. 
2. S. Helgason, $A$ duality in integral geometry on symmetric spaces, Proc. U. S.-Japan Seminar in Differential Geometry (Kyoto, 1965), Nippon Hyoronsha, Tokyo, 1966, pp. 3756. MR $37 \# 4765$.

3. - Differential geometry and symmetric spaces, Academic Press, New York, 1962. MR 26 \#2986.

4. T. O. Sherman, Fourier analysis on the sphere, Trans. Amer. Math. Soc. 209 (1975), 1-31.

5. R. J. Stanton, Mean convergence of fourier series on compact Lie groups, Trans. Amer. Math. Soc. 218 (1976), 61-87.

DEPARTMENT OF MATHEMATICS, NORTHEASTERN UNIVERSITY, BOSTON, MASSACHUSETTS 02115 